\title{
Capacidade anaeróbia em futebolistas de diferentes níveis competitivos: Comparação entre diferentes posições de jogo
}

\section{Anaerobic capacity in soccer players from different competitive levels: Comparison of players in different field positions}

\author{
V.L. Moro, K. Fuke, L. Cancian, S.C. Matheus, A.R. Moro
}

ARTIGO ORIGINAL | ORIGINAL ARTICLE

\begin{abstract}
RESUMO
O objetivo do presente estudo foi comparar a capacidade anaeróbia em jogadores profissionais de futebol de diferentes níveis competitivos e em suas respectivas posições de jogo, no início da pré-temporada. O grupo de estudo foi constituído por 44 atletas, do sexo masculino, pertencentes a dois grupos (A e B) de níveis competitivos diferentes. O grupo A foi composto por 20 atletas da $1^{\text {a }}$ divisão do Campeonato Português. O grupo B foi composto por 24 atletas da $2^{\text {a }}$ divisão do Campeonato Gaúcho - Brasil. Para a avaliação da capacidade anaeróbia foi utilizado o Running-based Anaerobic Sprint Test - RAST. Para análise dos dados foi utilizado o teste $t$ de Student para amostras independentes e análise de variância (ANOVA One Way) completada com o post hoc de Tukey. Os resultados mostram que os atletas do grupo A apresentaram melhor desempenho referente à potência média e piores valores para o índice de fadiga. No grupo A foram encontradas diferenças estatísticas entre atacantes e outras duas posições (meio-campistas e laterais) para o índice de fadiga. Concluímos que existem diferenças significativas na capacidade anaeróbia, avaliada no início da pré-temporada, entre atletas de futebol que atuam em competições de diferentes níveis e posições de jogo (grupo A).

Palavras-chave: futebol, atletas, capacidade anaeróbia, índice de fadiga
\end{abstract}

ABSTRACT

The aim of this study was to compare anaerobic capacity in professional soccer players of different competitive levels in their respective positions in the game, early in the preseason. The study group consisted of 44 male athletes belonging to two groups (A and B) of different competitive levels. Group A was composed of 20 athletes participating in the Sagres League - Portuguese. Group B was composed of 24 athletes participating in the 2nd division Championship Gaucho - Brazil. For the assessment of anaerobic capacity it was applied the Running-based Anaerobic Sprint Test - RAST. For data analysis it was used the Student $t$ test for independent samples and analysis of variance (ANOVA One Way) supplemented with post hoc Tukey. Results showed that group A athletes performed better on the average power and worst values for the fatigue index. In the group A it was found statistical difference between attacks and other two positions (midfielder and side back) for the fatigue index. It is concluded that there was significant differences in anaerobic capacity, measured at the start of the preseason, evaluated soccer players who work in competitions of different levels and positions play (group A).

Keywords: soccer, athletes, anaerobic capacity, fatigue index

Submetido: 10.06 .2011 | Aceite: 29.08.2012

Vanderson Luis Moro e Antonio Renato Pereira Moro. Universidade Federal de Santa Catarina - Brasil.

Kenji Fuke. Universidade Técnica de Lisboa - Portugal.

Lucas Cancian e Silvana Corrêa Matheus. Universidade Federal de Santa Maria - Brasil.

Endereço para correspondência: Vanderson Luis Moro. Laboratório de Cineantropometria, Centro de Educação Física e Desportos, Universidade Federal de Santa Maria. Avenida Roraima, 1000 Cidade Universitária,

Prédio 51, Bairro Camobi, Santa Maria, Rio Grande do Sul, Brasil, CEP 97.105-900, RS, Brasil.

E-mail: vandersonmoro@hotmail.com 
O futebol é uma modalidade desportiva extremamente complexa, de caráter intermitente, acíclico, com constantes mudanças de intensidade e função. Os praticantes dessa modalidade utilizam fontes energéticas distintas e possuem características fisiológicas peculiares (Bansgbo, 1994; Barbero, Villanueva, \& Bishop, 2006; Mohr, Krustrup, \& Bangsbo, 2003; Santos \& Soares, 2001; Stølen, Chamari, Castagna, \& Wisloff, 2005).

Conforme Reilly, Bangsbo e Franks (2000), os exercícios sem a bola compõem a maior parte do tempo de uma partida de futebol e, por isso, as demandas metabólicas predominantes durante um jogo de futebol são aeróbios. Entretanto, para esses mesmos autores as atividades decisivas no jogo são predominantemente anaeróbias.

Devido à realização repetida de esforços curtos e intensos, Stølen, Chamari, Castagna, e Wisloff (2005) afirmam que a potência anaeróbia é uma importante via de produção energética para os atletas de futebol, pois converte rapidamente a energia química em energia mecânica. Assim, esta conversão de energia é fundamental para potencializar a velocidade de deslocamento e ações de curta duração e alta intensidade (chutes, saltos e sprints).

Cada vez mais o trabalho de preparação física do futebolista de alto rendimento está direcionado para otimizar o metabolismo anaeróbio (Ostojic, 2004). Um rendimento eficiente deste metabolismo implica na manutenção, durante o maior tempo possível, da execução de esforços realizados a máxima velocidade, sem que haja um decréscimo prejudicial ao nível de desempenho (Davis \& Brewer, 1993).

Um dos testes de campo comumente utilizado pelos profissionais que trabalham com futebol para avaliar o desempenho anaeróbio (metabolismo alático e lático) é o Running-based Anaerobic Sprint Test - RAST (Zacharogiannis, Paradisis, \& Tziortzis, 2004). Tal teste fornece aos técnicos e treinadores medidas de potência e de índice de fadiga, sendo mais espe- cífico para os desportos em que o padrão básico de movimento é a corrida (Zagatto, Beck, \& Gobatto, 2009), tal como o futebol.

A avaliação da capacidade anaeróbia no período preparatório (pré-temporada) de jogadores de futebol é importante para o diagnóstico, controle e prescrição do treino, uma vez que é nesta época que os atletas adquirem ou restabelecem a forma desportiva para as fases iniciais da competição (Pinasco \& Carson, 2005). Além disso, tal avaliação permite programar-se em relação ao tempo de recuperação, tempo e modelo de trabalho, diminuindo cada vez mais a subjetividade dos treinamentos físicos (Rosell, Tarrero, Sassi, Marco, \& Cifre, 2000).

A pré-temporada da maioria das equipes de futebol é realizada em um período curto de treinamento ( \pm 40 dias), sendo que no final deste período os atletas devem estar preparados para um alto nível de competitividade. Por isso, a avaliação funcional no início da pré-temporada é importante para verificar a condição física e as potencialidades iniciais dos atletas. Pois, dependendo dos resultados dos testes fisiológicos realizados no início da pré-temporada, é possível adiantar fases de treinamento que seriam desenvolvidas somente após a competição já ter sido iniciada.

O desempenho anaeróbio vem sendo investigado em futebolistas de diferentes níveis competitivos e posições de jogo (Impellizzeri et al., 2008; Mohr et al., 2003). Segundo Mohr, Krustrup, e Bangsbo (2003), os atletas de elite apresentam maior desempenho nas atividades de alta intensidade, demonstrando que estes executam $58 \%$ mais sprints que atletas de sub-elite. Nesse mesmo estudo foi observado que apenas os goleiros apresentaram uma performance de corrida em alta intensidade menor que os zagueiros, meio-campistas e atacantes.

A comparação do desempenho anaeróbio entre as diferentes posições de jogo torna-se importante para identificar as características específicas de cada posição, maximizando os 
treinos em função de suas especificidades.

A comparação do desempenho anaeróbio em jogadores de futebol é capaz de distinguir atletas de diferentes níveis competitivos, sendo um fator limitante na ascensão profissional do futebolista (Gall, Carling, Williams, \& Reilly, 2010). Neste contexto, o conhecimento do desempenho anaeróbio de atletas de futebol de elite serve como parâmetro para atletas que almejam competir em níveis mais elevados.

Por outro lado, atletas que competem em níveis mais baixos podem aprimorar o desempenho anaeróbio, buscando adequar-se as exigências físicas de competições mais elevadas, uma vez que são comuns equipes de elite buscarem atletas em divisões inferiores ou em outros países. Por exemplo, a partir da análise de dados disponibilizados pela Confederação Brasileira de Futebol [CBF] (2010), Portugal foi o país que mais contratou atletas brasileiros de futebol no ano de 2008 (209 atletas) e no ano de 2009 (181 atletas), sendo que, na grande maioria, os atletas estavam vinculados a equipes de divisões inferiores no Brasil e se transferiram para equipes da $1^{\text {a }}$ divisão do Campeonato Português.

Com base nas informações anteriormente colocadas, o presente trabalho teve como objetivo principal comparar a capacidade anaeróbia em atletas profissionais de futebol de diferentes níveis competitivos e em suas respectivas posições de jogo, no início da pré-temporada.

\section{MÉTODO}

\section{Amostra}

O grupo de estudo foi constituído por 44 atletas profissionais de futebol de campo, do sexo masculino, pertencentes a equipes de diferentes níveis competitivos. O grupo A foi composto por 20 atletas profissionais de elite, todos com naturalidade brasileira que participavam da $1^{\text {a }}$ divisão da Liga Sagres Portugal, na temporada 2008/2009. O grupo B foi composto por 24 atletas profissionais de sub-elite, todos brasileiros participantes da $2^{\text {a }}$ divisão do Campeonato Gaúcho - Brasil, durante a temporada de 2009. Os goleiros de ambos os grupos foram excluídos do presente estudo por realizarem ações de curta duração e alta intensidade diferentes dos demais atletas de futebol durante o jogo, tais como defesas em chutes e cabeçadas a gol, saídas da meta para interceptação de lançamentos, cruzamentos e reposições de bola em jogo, além de realizar de forma passiva sua recuperação pós-esforço.

A constituição do grupo foi devido ao grande interesse de clubes portugueses por futebolistas brasileiros. Segundo dados da CBF (2010), Portugal foi o país que mais contratou atletas brasileiros de futebol no ano de 2008 (209 atletas) e no ano de 2009 (181 atletas).

Mesmo atuando em regiões demográficas diferentes, no momento da coleta de dados, ambos os grupos encontravam-se no início da pré-temporada, retornando de um período de destreinamento de 30 dias (férias). Anterior ao período de destreino, as duas equipes realizaram uma temporada de 10 meses. O nível de treinamento dos atletas, antes do período de férias, era similar entre as equipes, sendo realizado em torno de quatro a seis sessões semanais de treinos, com duração de aproximadamente 90 minutos por sessão e entre um e dois jogos oficiais por semana.

Cabe ressaltar que o nível de treinamento dos atletas de futebol depende do desempenho de suas respectivas equipes durante a temporada, ou seja, à medida que as equipes avançam de fase no campeonato que estão disputando, o número de jogos por semana será maior, acarretando a diminuição das sessões de treino e vice-versa. Essa variação de jogos e sessões de treino entre os grupos (A e B) pode ter interferido nos resultados do presente estudo.

\section{Instrumentos e Procedimentos}

Inicialmente os participantes foram informados a respeito dos propósitos e dos procedimentos a serem adotados durante a investigação. Os atletas profissionais de futebol, de 
ambos os grupos, conheciam os procedimentos e estavam familiarizados com a avaliação da capacidade anaeróbia (RAST), uma vez que a avaliação desta variável é realizada frequentemente pelos preparadores físicos. Após a concordância em participar do estudo, os atletas foram submetidos a avaliações antropométricas e, em seguida, realizaram o teste de capacidade anaeróbia.

$\mathrm{O}$ estudo seguiu as normas de pesquisa envolvendo seres humanos estabelecidos pela declaração de Helsinki e diretrizes da resolução 196/96, do Conselho Nacional de Saúde.

As medidas antropométricas foram obtidas por meio de uma balança mecânica da marca Welmy com estadiômetro acoplado, capacidade de $150 \mathrm{~kg}$ e precisão de 100 gr para a massa corporal e $210 \mathrm{~cm}$ de altura com resolução de $0.5 \mathrm{~cm}$ para mensurar a estatura. Para a obtenção dessas medidas seguiu-se os procedimentos descritos pela International Society for the Advancement of Kinanthropometry (2001).

Para a avaliação da capacidade anaeróbia, foi utilizado o RAST (Zacharogiannis et al., 2004). Tal teste foi validado recentemente no Brasil (Zagatto, Beck, \& Gobatto, 2009), apresentando correlação significativa $(p<.05)$ com o Wingate Test (potência média: $r=.53$ e índice de fadiga: $r=.63$ ).

O RAST consiste na realização de seis corridas de $35 \mathrm{~m}$ em velocidade máxima, com intervalo de 10 segundos de recuperação entre as corridas. O tempo foi registrado por meio de um sistema de fotocélulas (Multi Sprint, Hidrofit ${ }^{\circledR}$, Belo Horizonte-MG, Brasil), dispostas a $35 \mathrm{~m}$ de distância, para capturar os tempos dos sprints.

A partir dos dados coletados foram calculadas a potência média e o índice de fadiga.

\section{Análise Estatística}

Os dados foram analisados estatisticamente por meio de análises descritivas, que compreenderam valores de média e desvio padrão. A normalidade dos dados foi confirmada pelo teste de Shapiro Wilk. A análise entre cada posição de jogo de atletas do grupo A e B foi feita por meio do teste $t$ de Student para amostras independentes. A comparação entre as posições de jogo dos atletas do mesmo nível competitivo foi realizada por meio da análise de variância (ANOVA One Way) completada com o post hoc de Tukey. O nível de confiança adotado foi de $95 \%$. Utilizou-se o programa estatístico GraphPad Prism 5.

\section{RESULTADOS}

Os dados referentes à caracterização dos atletas avaliados estão expressos na Tabela 1 por meio de valores de média e desvio-padrão.

A comparação da potência média e do índice de fadiga entre o grupo A ( $1^{\text {a }}$ divisão do Campeonato Português) e o grupo B ( $2^{\text {a }}$ divisão do Campeonato Gaúcho/Brasil) de acordo com a posição de jogo são apresentados nas Figuras 1 e 2 , respectivamente.

Ao verificar os valores da potência média (Figura 1) constata-se que o grupo A apresentou valores maiores e estatisticamente significativos em comparação com a equipe B para a maioria das posições de jogo investigadas (zagueiros, laterais e meio-campistas). Apenas os atacantes não diferiram estatisticamente entre os dois grupos de estudo para esta mesma variável.

Os resultados referentes ao índice de fadiga (Figura 2) dos atletas de futebol de acordo com a sua posição de jogo mostram maiores valores deste índice para o grupo A. Entretanto, apenas os meio-campistas diferiram estatisticamente entre os grupos (A e B).

A comparação da potência média e do índice de fadiga entre as diferentes posições de jogo dentro de cada grupo pode ser observado nas Figuras 3 e 4, respectivamente.

Na Figura 3, pode ser observado que a potência média não diferiu estatisticamente entre as posições de jogo para atletas de futebol do mesmo nível competitivo, independente do grupo investigado (A ou B). 
Tabela 1

Caracterização dos atletas de acordo com as posições de jogo

\begin{tabular}{ccccc}
\hline Grupo A & Zagueiros & Laterais & Meio-campistas & Atacantes \\
$n=5$ & $n=4$ & $n=6$ & $n$ \\
\hline Idade (anos) & $26.9 \pm 3.5$ & $24.8 \pm 6.1$ & $29.3 \pm 4.6$ & $25.2 \pm 4$ \\
Massa corporal (Kg) & $82.1 \pm 8.1$ & $72.9 \pm 5.2$ & $76.4 \pm 5.5$ & $80.2 \pm 7.5$ \\
Estatura (cm) & $183.4 \pm 4.3$ & $173.5 \pm 7.1$ & $182.8 \pm 6.9$ & $181.8 \pm 9.8$ \\
Tempo de prática (anos) & $13.3 \pm 4.1$ & $12.6 \pm 5.7$ & $15.1 \pm 6.8$ & $13.1 \pm 3.3$ \\
\hline Grupo B & Zagueiros & Laterais & Meio-campistas & Atacantes \\
& $n=5$ & $n=5$ & $n=9$ & $n=5$ \\
\hline Idade (anos) & $27.7 \pm 4.1$ & $25.5 \pm 6.1$ & $27.9 \pm 6.6$ & $26.2 \pm 3.7$ \\
Massa corporal (Kg) & $82.9 \pm 3.8$ & $68.7 \pm 6.9$ & $76.1 \pm 5.3$ & $78.9 \pm 3.8$ \\
Estatura (cm) & $180.5 \pm 5.1$ & $173.6 \pm 5.1$ & $175 \pm 6.3$ & $183.2 \pm 4.8$ \\
Tempo de prática (anos) & $13.6 \pm 5.4$ & $12.1 \pm 4.9$ & $14.2 \pm 6.2$ & $12.7 \pm 3.3$ \\
\hline
\end{tabular}

Potência média

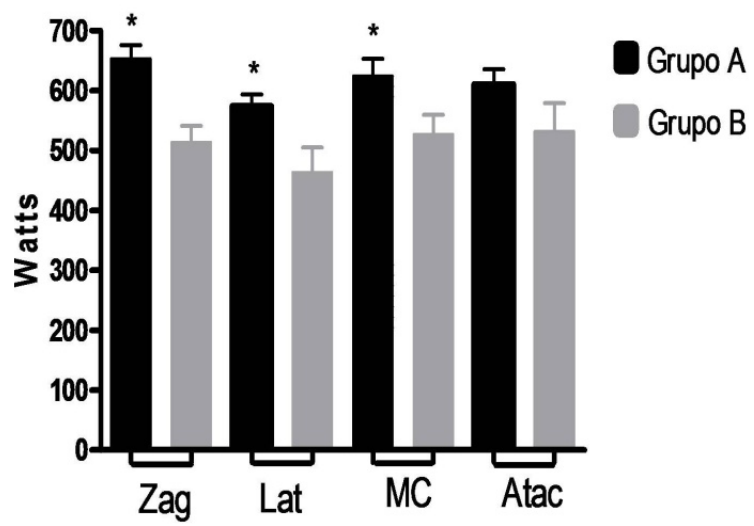

Figura 1. Valores da potência média dos atletas de futebol dos grupos A ( $1^{\text {a }}$ divisão do Campeonato Português) e B ( $2^{\text {a }}$ divisão do campeonato Gaúcho/ Brasil) separados por posições de jogo. Zag (Zagueiros), Lat (Laterais), MC (Meio-campistas) e Atac (Atacantes). Cada coluna representa a média \pm dp dos atletas. (Teste " $t$ " de Student para amostras independentes) $* p<.05$.

O índice de fadiga (Figura 4) não diferiu estatisticamente entre as posições de jogo para atletas de futebol do grupo B. Já o grupo A apresentou valores menores e estatisticamente significativos para os laterais na comparação com os laterais e meio-campistas.

\section{DISCUSSÃO}

Em geral, os resultados mostram que os atletas brasileiros que disputam competições
Índice de fadiga

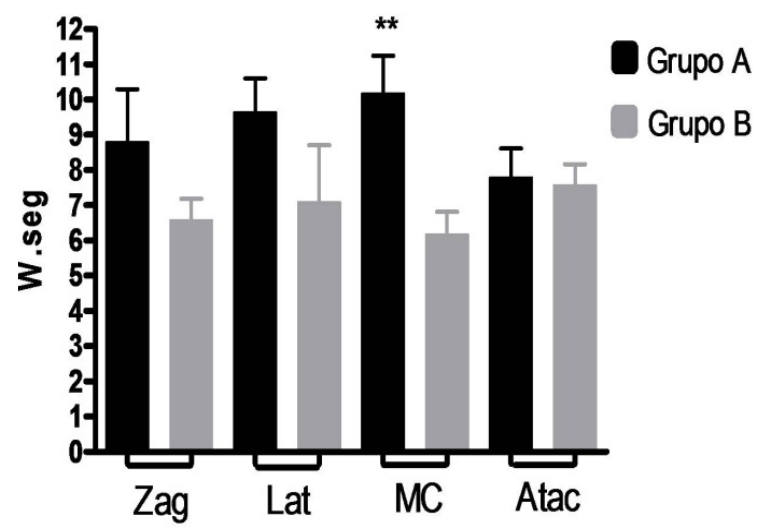

Figura 2. Valores do índice de fadiga dos atletas de futebol dos grupos A e B separados por posições de jogo. Zag (Zagueiros), Lat (Laterais), MC (Meio-campistas) e Atac (Atacantes). Cada coluna representa a média $\pm \mathrm{dp}$ dos atletas. (Teste " $t$ " de Student para amostras independentes) ${ }^{* *} p<.001$.

em níveis mais elevados (grupo A) apresentaram melhores resultados referentes à potência média (Figura 1) e piores valores para o índice de fadiga (Figura 2). Outro achado importante do presente estudo foi que na comparação entre as diferentes posições de jogo no mesmo nível competitivo, não foi possível identificar a especificidade de cada posição de jogo quanto ao desempenho da potência média, no início da pré-temporada (Figura 3). No entanto, o índice 
Potência média

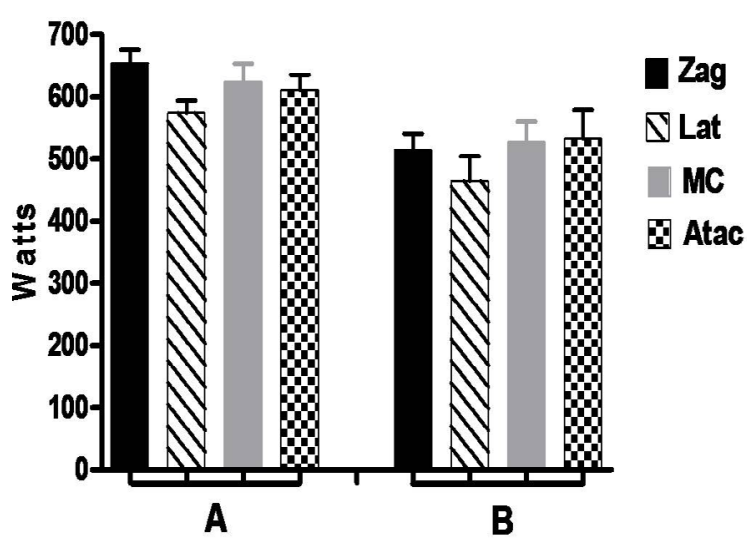

Figura 3. Valores da potência média dos atletas de futebol da equipe A e B separados por posições de jogo. Zag (Zagueiros), Lat (Laterais), MC (Meio-campistas) e Atac (Atacantes). Cada coluna representa a média $\pm \mathrm{dp}$ dos atletas. (ANOVA One Way).

de fadiga (Figura 4) dos atacantes da equipe $\mathrm{A}$ diferiu dos meio-campistas e laterais, no início do período preparatório.

A avaliação da capacidade anaeróbia dos atletas foi realizada no início da pré-temporada de cada equipe. A pré-temporada dos atletas participantes da $1^{\text {a }}$ divisão da Liga Sagres Portugal teve início no mês de julho (verão europeu) e dos atletas participantes $2^{\text {a }}$ divisão do Campeonato Gaúcho - Brasil iniciou-se no mês de janeiro (verão brasileiro). No período da coleta de dados a estação do ano era a mesma em ambos os países, no entanto as condições climatéricas (temperatura, umidade e velocidade do vento) que eventualmente podem influenciar nos resultados não foram mensuradas, sendo isto uma limitação do presente estudo.

Muitos estudos (Abrantes, Maçãs, \& Sampaio, 2004; Al-Hazzaa et al., 2001; Aziz, Mukherjee, Chia, \& Teh, 2007, 2008; Cruz, 2005; McIntyre \& Hall, 2005; Kaplan, 2010; Ribeiro, Dias, Claudino, \& Gonçalves, 2007) realizados com atletas de futebol utilizam diferentes protocolos para avaliação da capacidade anaeróbia, tais como Wingate Test, Bangsbo Repeated Sprint Test, Running Repeated Sprint
Índice de fadiga

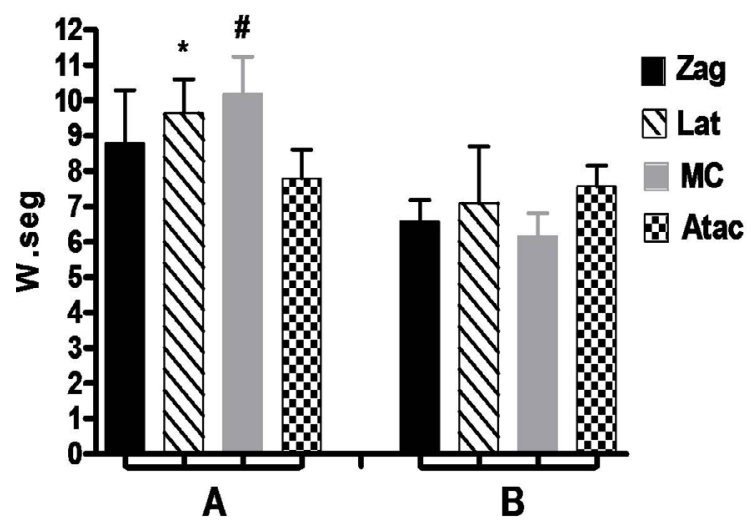

Figura 4. Valores do índice de fadiga dos atletas de futebol do grupo A e B separados por posições de jogo. Zag (Zagueiros), Lat (Laterais), MC (Meio-campistas) e Atac (Atacantes). Cada coluna representa a média $\pm \mathrm{dp}$ dos atletas. Símbolo “*” representa diferença entre laterais $\mathrm{x}$ atacantes. Símbolo “\#” representa diferença entre meio-campistas x atacantes (ANOVA One Way confirmadas com post hoc de Tukey) $p<.05$.

Ability Test, Running-based Anaerobic Sprint Test, entre outros. Porém, é necessário ter cautela na comparação entre tais protocolos, uma vez que gera um grande número de índices de desempenho anaeróbio com pressupostos de base diferentes.

No entanto, independente do protocolo utilizado, a maioria dos estudos mostra diferenças estatísticas nos indicadores de desempenho anaeróbio entre atletas profissionais de elite ( $1^{\text {a }}$ divisão de campeonatos nacionais e internacionais) e amadores (Cometti, Maffiuletti, Pousson, Chatard, \& Maffulli, 2001; Impellizzeri et al., 2008), atletas profissionais de sub-elite (divisões inferiores) e amadores (Cometti et al., 2001), semi-profissionais (atletas jovens) e amadores (Aziz et al., 2008).

Abrantes, Maçãs, e Sampaio (2004) utilizaram o Bangsbo Repeated Sprint Test para investigar o tempo médio de sprints em atletas de futebol de diferentes níveis competitivos. Os autores encontraram diferença estatística entre todos os grupos (atletas profissionais de elite, sub-elite e amadores) e concluíram que os atletas profissionais de elite apresentam melhor 
desempenho em testes de sprints, quando comparados aos atletas de níveis competitivos inferiores.

Assim, os resultados da capacidade anaeróbia encontrados no presente estudo corroboram os estudos anteriormente citados, evidenciando que o desempenho da capacidade anaeróbia melhora à medida que o nível competitivo aumenta.

Em contrapartida, foi encontrado na literatura apenas um estudo que compara a mesma posição de jogo em níveis competitivos diferentes. Ribeiro, Dias, Claudino, e Gonçalves (2007), utilizando o mesmo protocolo de teste do presente estudo (RAST), não encontraram diferença estatística ao comparar a potência média de jovens atletas brasileiros de futebol profissional e semi-profissional. Esses autores compararam apenas uma posição de jogo (laterais/alas) entre os diferentes níveis competitivos. Possivelmente, os autores não encontraram diferença entre os dois níveis competitivos devido à idade (18.4 \pm 0.7 anos) do grupo de estudo, pois nesta faixa etária os atletas encontram-se em fase de especialização física na modalidade (Marques, Travassos, \& Almeida, 2010) e os índices de desempenho desportivo, entre eles, o desempenho anaeróbio, estão correlacionados com o nível de maturação (Villar \& Denadai, 2001).

A potência média no presente estudo não diferiu estatisticamente entre as posições de jogo para atletas de futebol do mesmo nível competitivo, independente do grupo investigado (A ou B). Davis, Brewer, e Atkin (1992) realizaram o Wingate test em atletas de futebol no início da temporada da liga inglesa e apresentaram resultados semelhantes aos do presente estudo.

No entanto, Al-Hazzaa et al. (2001) avaliaram atletas da seleção nacional da Arábia Saudita no início da fase de preparação para a copa do mundo da França (1998) e mostraram que a potência média dos zagueiros apresentou valores maiores e estatisticamente significa- tivos em comparação com atletas de outras posições de jogo (laterais, meio-campistas e atacantes). No estudo de Cruz (2005), com atletas portugueses que atuavam na $1^{\text {a }}$ divisão do campeonato Português e estavam em início da pré-temporada, também foram encontradas diferenças estatisticamente significativas para a potência média. Neste estudo, os atacantes apresentaram valores maiores e estatisticamente significativos na comparação com os meio-campistas, independentemente do protocolo de teste utilizado (RAST ou Wingate test).

Com base nos trabalhos anteriormente citados, relacionados à potência média em futebolistas, fica evidente a necessidade de realizar mais estudos que caracterizem cada posição de jogo, pois não houve um consenso entre os trabalhos que analisaram esta variável em atletas de futebol do mesmo nível competitivo.

Outra variável investigada no presente estudo foi o índice de fadiga. Esta apresentou valores inferiores no grupo B em comparação ao grupo A (Figura 2), porém, apenas os meio-campistas diferiram estatisticamente entre os grupos. De acordo com Zacharogiannis et al. (2004) os valores do índice de fadiga recomendados para atletas de futebol são de até $10 \%$, sendo que valores acima indicam a necessidade de melhorar a tolerância aos esforços intermitentes. No entanto, deve-se ter cautela na utilização do índice de fadiga, pois no cálculo desse índice no RAST consideram-se apenas a maior e a menor potência e não o decréscimo da potência nos seis sprints. Os atletas da equipe B estavam com a potência anaeróbia máxima muito baixa e, consequentemente, a diferença entre a potência máxima e mínima foi menos significativa. Isso pode ter influenciado os resultados do índice de fadiga apresentados no presente estudo, pois quanto menor é a potência, parece ser mais fácil de mantê-la.

Kaplan (2010) investigou o índice de fadiga em 85 atletas turcos amadores de futebol, de mesmo nível competitivo, de acordo com as posições de jogo. Os atletas foram avaliados no 
final do período preparatório (após 6 semanas de treinamento). O protocolo utilizado para obter os valores de índice de fadiga foi o Bangsbo Repeated Sprint Test, similar ao teste de RAST. O autor não encontrou diferença estatística entre as posições de jogo.

Estudos realizados no início da pré-temporada, com atletas brasileiros semi-profissionais (Goulart, Dias, \& Altimari, 2007; Silva, Guglielmo, Floriano, Arins, \& Dittrich, 2009) e portugueses profissionais de elite (Cruz, 2005) também não encontraram diferença estatística entre as posições de jogo. Cruz (2005) avaliou o índice de fadiga em atletas portugueses profissionais de elite, por meio de dois protocolos distintos (teste de RAST e Wingate test), no mesmo período de treinamento (início da pré-temporada) e não encontrou diferença estatística entre as posições de jogo.

Os achados destes estudos estão de acordo com os encontrados no presente estudo para o grupo B e divergem do grupo A. Tal divergência do grupo A com esses estudos podem ser explicados pela diferença entre os níveis competitivos (Kaplan, 2010) e faixa etária (Goulart et al., 2007 e Silva et al., 2009), mostrando que, em níveis mais elevados de competições, pode ser possível identificar a especificidade da posição de jogo. No entanto, como mencionado anteriormente, existe uma limitação na utilização do cálculo do índice de fadiga (Figura 1) no RAST. Isso pode ter influenciado os resultados deste índice, devendo ser considerado como uma limitação do presente estudo.

$\mathrm{Na}$ comparação do índice de fadiga entre as posições de jogo, no mesmo nível competitivo, foram encontradas diferenças estatísticas entre atacantes e outras duas posições (meio-campistas e laterais) no grupo A (Figura 4). Esses resultados indicam uma possível especificidade da posição de jogo. No entanto, mais estudos devem ser realizados com atletas de futebol para comprovar estes achados, enfatizando as causas da fadiga muscular relacionada ao desempenho anaeróbio em diferentes posições de jogo e em níveis competitivos mais elevados.

Para tanto, é importante ressaltar que a fadiga muscular pode ser causada por diversos fatores, tais como o calor, a umidade atmosférica, a desidratação, a redução da funcionalidade do sistema nervoso central, a depleção de substratos, entre outros (Mohr, Krustrup, \& Bangsbo, 2005), sendo que a fadiga muscular no final de uma partida de futebol parece ser principalmente em decorrência da depleção do glicogênio muscular em algumas fibras musculares (Bangsbo, Iaia, \& Krustrup, 2007; Mohr et al., 2005).

Bangsbo, Iaia, e Krustrup (2007) apresentam, em uma revisão de estudos sobre a fadiga no futebol, afirmando que o glicogênio muscular é o substrato mais importante para a produção de energia, uma vez que há uma redução do glicogênio muscular entre $40 \mathrm{e}$ $90 \%$ após uma partida de futebol. Entretanto, no presente estudo a depleção do glicogênio muscular não deve ter sido o principal responsável pelos elevados índices de fadiga no grupo A, pois a fadiga temporária ocasionada por períodos intensos e de curta duração durante o jogo, similar ao teste de RAST, está atrelado a distúrbios na homeostasia iônica do músculo e a diminuição da excitabilidade do sarcolema (Mohr et al., 2005) e estas parecem ter sido as razões dos elevados índices de fadiga no grupo A.

\section{CONCLUSÕES}

Com base nos resultados encontrados, é possível concluir que atletas de futebol (zagueiros, meio-campistas e atacantes) que competem em níveis mais elevados apresentam melhor desempenho da capacidade anaeróbia, avaliada no início da pré-temporada, em comparação com atletas de futebol de níveis competitivos inferiores. Esses achados indicam que atletas de futebol de níveis competitivos mais elevados necessitam um maior treinamento da capacidade anaeróbia para suportar as exigências de competições de alto rendi- 
mento. A comparação do índice de fadiga entre atletas que competem em níveis mais elevados mostra que os atacantes apresentam melhores valores do que outras duas posições de jogo (meio-campistas e laterais). Isso indica que os atacantes apresentam maior tolerância aos esforços intermitentes, possibilitando aos preparadores físicos que enfatizem mais o treinamento anaeróbio nesses atletas. Sugere-se que sejam realizados estudos comparando atletas de futebol que competem em diferentes países realizando um acompanhamento do desempenho anaeróbio durante toda a temporada. Além de utilizar o decréscimo da potência dos seis sprints (RAST) para análise da fadiga muscular.

\section{Agradecimentos:}

Nada declarado.

\section{Conflito de Interesses:}

Nada declarado.

\section{Financiamento:}

Nada declarado.

\section{REFERÊNCIAS}

Abrantes, C., Maçãs, V., \& Sampaio, J. (2004). Variation in football players sprint test performance across different ages and levels of competition. Journal of Sports Science and Medicine, 3(1), 44-49.

Al-Hazzaa, H. M., Almuzaini, K. S., Al-Refafe, S. A., Sulaiman, M. A., Dafterdar, M. Y., Al-Ghamedi, A., \& Al-Khuraiji, K. N. (2001). Aerobic and anaerobic power characteristics on Saudi elite soccer players. Journal of Sports Medicine and Physical Fitness, 41 (1), 54-61.

Aziz, A. R., Mukherjee, S., Chia, M. Y. H., \& Teh, K. C. (2007). Relationship between measured maximal oxygen uptake and aerobic endurance performance with running repeated sprint ability in young elite soccer players. Journal of Sports Medicine and Physical Fitness, 47(4), 401-407.

Aziz, A. R., Mukherjee, S., Chia, M. Y. H., \& Teh, K. C. (2008). Validity of the running repeated sprint ability test among playing positions and level of competitiveness in trained soccer players. Inter- national Journal Sports Medicine, 29(9), 833-838.

Bangsbo, J. (1994). The physiology of soccer: With special reference to intense intermittent exercise. Acta Physiologica Scandinavica, 151 (suppl.), 619.

Bangsbo, J., Iaia, F. M., \& Krustrup, P. (2007). Metabolic response and fatigue in soccer. International Journal of Sports Physiology and Performance Journal, 2(2), 111-127.

Barbero, J. C., Villanueva, A. M., \& Bishop, D. (2006). La capacidad para repetir esfuerzos máximos intermitentes: aspectos fisiológicos (I). Archivos de Medicina del Deporte, 23(114), 299-303.

Confederação Brasileira de Futebol. Relação de transferências para o exterior. Recuperado em 14 de novembro, 2010, de http://www.cbf.com.br/ php/transferencias.php

Cometti, G., Maffiuletti, N. A., Pousson, M., Chatard, J. C., \& Maffulli, N (2001). Isokinetic strength and anaerobic power of elite, subelite and amateur French soccer players. International Journal Sports Medicine, 22 (1), 45-51.

Cruz, S. (2005). Avaliação da capacidade física em futebolistas profissionais: Análise em função da posição específica. (Dissertação de Mestrado não-publicada), Universidade do Porto, Faculdade de Ciências do Desporto e de Educação Física, Portugal.

Davis, J. A., \& Brewer, J. (1993). Applied physiology of female soccer players: Review. Sports Medicine, 16(3), 180-189.

Davis, J. A., Brewer, J., \& Atkin, D. (1992). Pre-season physiological characteristics of English first and second division soccer players. Journal of Sports Sciences, 10(6), 541-547.

Gall, F. L., Carling, C., Williams, M., \& Reilly, T. (2010). Anthropometric and fitness characteristics of international, professional and amateur male graduate soccer players from an elite youth academy. Journal of Science and Medicine in Sport, 13(1), 90-95.

Goulart, L. F., Dias, R. M. R., \& Altimari, L. R. (2007). Força isocinética de jogadores de futebol categoria sub-20: Comparação entre diferentes posições de jogo. Revista Brasileira de Cineantropometria e Desempenho Humano, 9(2), 165-169. 
Impellizzeri, F. M., Rampinini, E., Castagna, C., Bishop, D., Ferrari Bravo, D., Tibaudi, A., \& Wisloff, U. (2008). Validity of a repeated-sprint test for football. International Journal Sports Medicine, 29(11), 899-905.

International Society for the Advancement of Kinanthropometry. (2001). International standards for anthropometric assessment. (1st ed.). Adelaide: National Library of Australia Press.

Kaplan, T. (2010). Examination of repeated sprinting ability and fatigue index of soccer players according to their positions. The Journal of Strength and Conditioning Research, 24(6), 1495-1501.

Marques, M. C., Travassos, B., \& Almeida, R. (2010). A força explosiva, velocidade e capacidades motoras específicas em futebolistas juniores amadores: Um estudo correlacional. Motricidade, 6(3), 5-12.

McIntyre, M. C., \& Hall, M. (2005). Physiological profile in relation to playing position of elite college Gaelic footballers. British Journal of Sports Medicine, 39(5), 264-266.

Mohr, M., Krustrup, P., \& Bangsbo, J. (2003). Match performance of high-standard soccer players with special reference to development of fatigue. Journal of Sports Sciences, 21, 439-449.

Mohr, M., Krustrup, P., \& Bangsbo, J. (2005). Fatigue in soccer: A brief review. Journal of Sports Science, 23(6), 593-599.

Ostojic, S. M. (2004). Elite and nonelite soccer players: Preseasonal physical and physiological characteristics. Research in Sports Medicine, 12(2), 143-150.

Pinasco, A., \& Carson, J. (2005). Preseason conditioning for college soccer. Strength and Conditioning Journal, 27(5), 56-62.
Reilly, T., Bangsbo, J., \& Franks, A. (2000). Anthropometric and physiological predispositions for elite soccer. Journal of Sports Science, 18(9), 669-683.

Ribeiro, R. S., Dias, D. F., Claudino, J. G. O., \& Gonçalves, R. (2007). Análise do somatotipo e condicionamento físico entre atletas de futebol de campo sub-20. Motriz, 13(4), 280-287.

Rosell, J. C., Tarrero, L. M. T., Sassi, R., Marco, A. G., \& Cifre, J. S. (2000). El trabajo intermitente de alta intensidad: Experiencia de campo. Archivos de Medicina del Deporte, 17(77), 257-262.

Santos, P. J., \& Soares, J. M. (2001). Capacidade aeróbia em futebolistas de elite em função da posição específica no jogo. Revista Portuguesa de Ciências do Desporto, 1(2), 7-12.

Silva, J. F., Guglielmo, L. G. A., Floriano, L. T., Arins, F. B., \& Dittrich, N. (2009). Aptidão aeróbia e capacidade de sprints repetidos no futebol: Comparação entre as posições. Motriz, 15(4), 861-870.

Stølen, T., Chamari, K., Castagna, C., \& Wisloff, U. (2005). Physiology of Soccer: An Update. Sports Medicine, 35(6), 501-536.

Villar, R., \& Denadai, B. S. (2001). Efeitos da idade na aptidão física em meninos praticantes de futebol de 9 a 15 anos. Motriz, 7(2), 93-97.

Zacharogiannis, E., Paradisis, G., \& Tziortzis, S. (2004). An evalution of tests of anaerobic power and capacity. Medicine and Science in Sports and Exercise, 36(5), 116.

Zagatto, A. M., Beck, W. R., \& Gobatto, C. A. (2009). Validity of the running anaerobic sprint test for assessing anaerobic power and predicting short-distance performances. The Journal of Strength and Conditioning Research, 23(6), 1820-1827.

(cc)BY-NC Todo o conteúdo da revista Motricidade está licenciado sob a Creative Commons, exceto quando especificado em contrário e nos conteúdos retirados de outras fontes bibliográficas. 